Case Report

\title{
Infectious Endocarditis from Enterococcus faecalis Associated with Tubular Adenoma of the Sigmoid Colon
}

\author{
Emilly Caroline de Freitas Silva, Camila Ronchini Montalvão, and Simone Bonafé \\ Centro de Ensino Superior de Maringá (UNICESUMAR), Avenida Guedner, 1610 Jardim Aclimação, 87050-390 Maringá, PR, Brazil \\ Correspondence should be addressed to Emilly Caroline de Freitas Silva; emilly.carolaine@hotmail.com
}

Received 17 February 2017; Revised 15 May 2017; Accepted 19 June 2017; Published 7 August 2017

Academic Editor: Tomoyuki Shibata

Copyright (C) 2017 Emilly Caroline de Freitas Silva et al. This is an open access article distributed under the Creative Commons Attribution License, which permits unrestricted use, distribution, and reproduction in any medium, provided the original work is properly cited.

\begin{abstract}
Introduction. Enterococcus faecalis (E. faecalis), a constituent of the gut microbiota, can be associated with both colonic lesions and endocarditis. Since this microorganism is one of the endocarditis etiological agents, there is a need for greater study in regard to the association with endocarditis and colonic lesions. Case Presentation. This is the case description of a 53-year-old man with history of prolapse of the anterior mitral valve leaflet who was diagnosed with endocarditis by E. faecalis and treated with ampicillin and gentamicin. Upon investigation by colonoscopy, he was found to have a tubular adenoma with low grade dysplasia. Conclusion. There are a few descriptions in scientific literature of an association between endocarditis by $E$. faecalis and colonic lesions. However, further studies with significant correlation between the two pathologies are required, so that proper measures can be implemented in clinical practice.
\end{abstract}

\section{Introduction}

Little is known about the pathophysiology of the interactions between infective endocarditis caused by $E$. faecalis and intestinal neoplasia. A few descriptions in literature associate the presence of these bacteria with preexisting colonic lesions [1-5]. It is recognized and well established that there is a strong correlation between these two pathologies when the isolated bacteria are Streptococcus gallolyticus (S. gallolyticus), formerly known as Streptococcus bovis (S. bovis) [6,7].

In this context, the objective of this case study is to present clinical, diagnostic, and therapeutic data of a patient that developed endocarditis by $E$. faecalis with coinciding colonic lesions.

\section{Case Presentation}

A 53-year-old male with history of prolapse and discrete myxomatous degeneration of the anterior mitral valve leaflet, mild-to-moderate aortic insufficiency, and midsystolic mitral regurgitation presented fever, nocturnal hyperhidrosis, myalgia, and anorexia during a period of approximately 15 days, without significant weight loss.
An investigation to elucidate the origin of the fever was initiated. The hemogram showed discrete leukocytosis $\left(10^{5}\right.$ cells $\left./ \mathrm{ml}\right)$ and an increase of the inflammatory markers ESR $(98 \mathrm{~mm})$ and CRP $(97 \mathrm{~mm} / \mathrm{L})$. Serologies for cytomegalovirus, Epstein Barr virus, HIV, toxoplasmosis, and syphilis were all negative, as well as ANA and PPD, which was nonreactive.

Urinalysis presented with intense leukocyturia $(289.740 \mathrm{cell} / \mathrm{s} / \mathrm{ml})$ and urine culture showed the presence of Escherichia coli. After collecting 3 samples of blood for culture (BacT/Alert 3D, Biomerieux method), it was decided to start antibiotics for urinary tract infection (UTI), even though the patient had no UTI symptoms at the time. The patient denied being submitted to any invasive urinary or gastrointestinal tract procedures, like the placement of vesical catheters or surgery.

Despite the use of gentamicin for the asymptomatic UTI, the initial symptoms persisted. Approximately 10 days after the initial investigation, the patient evolved with Janeway lesion and the onset of new diastolic aortic and systolic mitral murmurs. A transesophageal echocardiogram was performed and it revealed a discrete $5 \mathrm{~mm}$ mass (vegetation) 


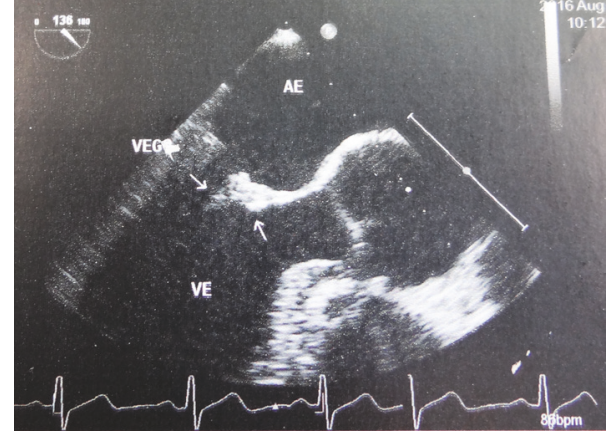

FIGURE 1: Vegetation on the anterior leaflet of the mitral valve shown on transesophageal echocardiogram.

on the anterior mitral valve leaflet (Figure 1). Furthermore, culture and antibiogram from all 3 previously collected blood samples revealed the presence of E. faecalis sensitive to ampicillin.

The patient was diagnosed with endocarditis and was subsequently hospitalized and prescribed ampicillin for a 28-day therapeutic cycle (due to a shortage of crystalline penicillin). Furthermore, gentamicin was extended for 14 days.

On the 23rd day of hospitalization a colonoscopy was solicited, given the patient's age, family history of colonic cancer, and scientific literature descriptions associating endocarditis with intestinal diseases. Intestinal preparation was duly performed and there was complete visualization of the colon up to the ileocecal valve (Figure 2). A $15 \mathrm{~mm}$ pedunculated polyp was visualized in the sigmoid colon and a polypectomy was performed. The final anatomopathological report determined tubular adenoma with low grade dysplasia.

The interval between the onset of symptoms and the conclusion of the case was approximately 2 months. The patient was released from the hospital following the end of the treatment with resolution of the infectious manifestations and, without complications, was referenced to follow-up cardiology care.

\section{Discussion}

Enterococcus is the third major cause of infectious endocarditis in the world, being responsible for $5 \%$ to $15 \%$ of cases. $E$. faecalis, responsible for the majority of these cases, is a minor commensal bacteria found in the intestinal microbiota [8].

The source of bacteremia by E. faecalis, in cases with cardiac infection as well as alterations in other systems, most often is undetermined. Research shows that up to $67.8 \%$ of bacteremia cases do not have an identifiable source [9, 10]. A frequent point of entrance, through which the $E$. faecalis invades the bloodstream, is the genitourinary tract, particularly in cases related to endocarditis. Contaminations through the gastrointestinal and biliary tracts, as well as catheters, are also cited in various studies $[1,9]$.

Possible associations between endocarditis by E. faecalis and colonic lesions have been demonstrated [1-5], although the data described in studies is insufficient to determine the magnitude and the meaning of this combination. Such an association is recognized with S. gallolyticus, being extensively documented in publications, even though the pathophysiological mechanism is not yet well elucidated [11].

These studies use a new nomenclature for what was previously known as $S$. bovis, $S$. bovis biotype I, and biotype II/2, which now correspond to S. gallolyticus and its subspecies. These microorganisms are mentioned in studies as being associated with colorectal cancer and with the development of bacteremia and endocarditis [11].

The latest discoveries, through genomic analysis, showed that S. gallolyticus has intrinsic characteristics capable of favoring its translocation, such as proteins that bind to collagen and the presence of 3 types of pili. These same characteristics, which make this microorganism highly proficient in causing bacteremia, also have the potential to cause endocarditis and intestinal lesions [12].

Regarding E. faecalis, it is believed that this agent is also involved in the mutation of colonic cells [13]. Some studies defend that the lesioned mucosa, be it due to ischemia or inflammation, is propitious to translocation of agents such as E. faecalis, leading to bacteremia $[2,9,14]$. It is known that the presence of a previous mitral prolapse predisposes to the onset of endocarditis caused by this microorganism.

Recent studies about inflammatory intestinal diseases and irritable bowel syndrome have pointed to potential mechanisms through which E. faecalis would be able to cause a dysfunction of the intestine's epithelial barrier [15]. However, even though some researchers have tried to show that in fact these bacteria found in the blood and in other sterile regions are originated from the gastrointestinal tract, there has been little progress and it has not yet been possible to comprehend how this species migrates [16]. Nonetheless, it is understood that the translocation of E. faecalis through healthy tissue is a rare event and has no damaging lasting repercussions $[17,18]$.

Regarding the case described in this study, the source of the infection was not identified. However, its simultaneity with a low grade intestinal dysplasia was observed, which can reinforce the hypothesis of E. faecalis being capable of translocating itself and causing endocarditis having, as a point of entry, a previous intestinal lesion. It is also relevant to emphasize that colorectal cancer, which originates from the same pathological process present in the patient being studied, is the fourth most prevalent neoplasia in the Brazilian male population, being relatively frequent in the age group of 50 years and older (INCA ESTIMATIVA 2016). Considering the fact that the patient here described is in the aforementioned gender and age group, such bias could be confounding in the sense of attributing the described mechanism to the endogenous intestinal E. faecalis. However, even with this limitation, this case description aggregates with others that have suggested the same supposed interaction [1-5].

\section{Conclusion}

The occurrence of endocarditis by E. faecalis of a nonidentifiable source is not uncommon, as seen with the frequency 

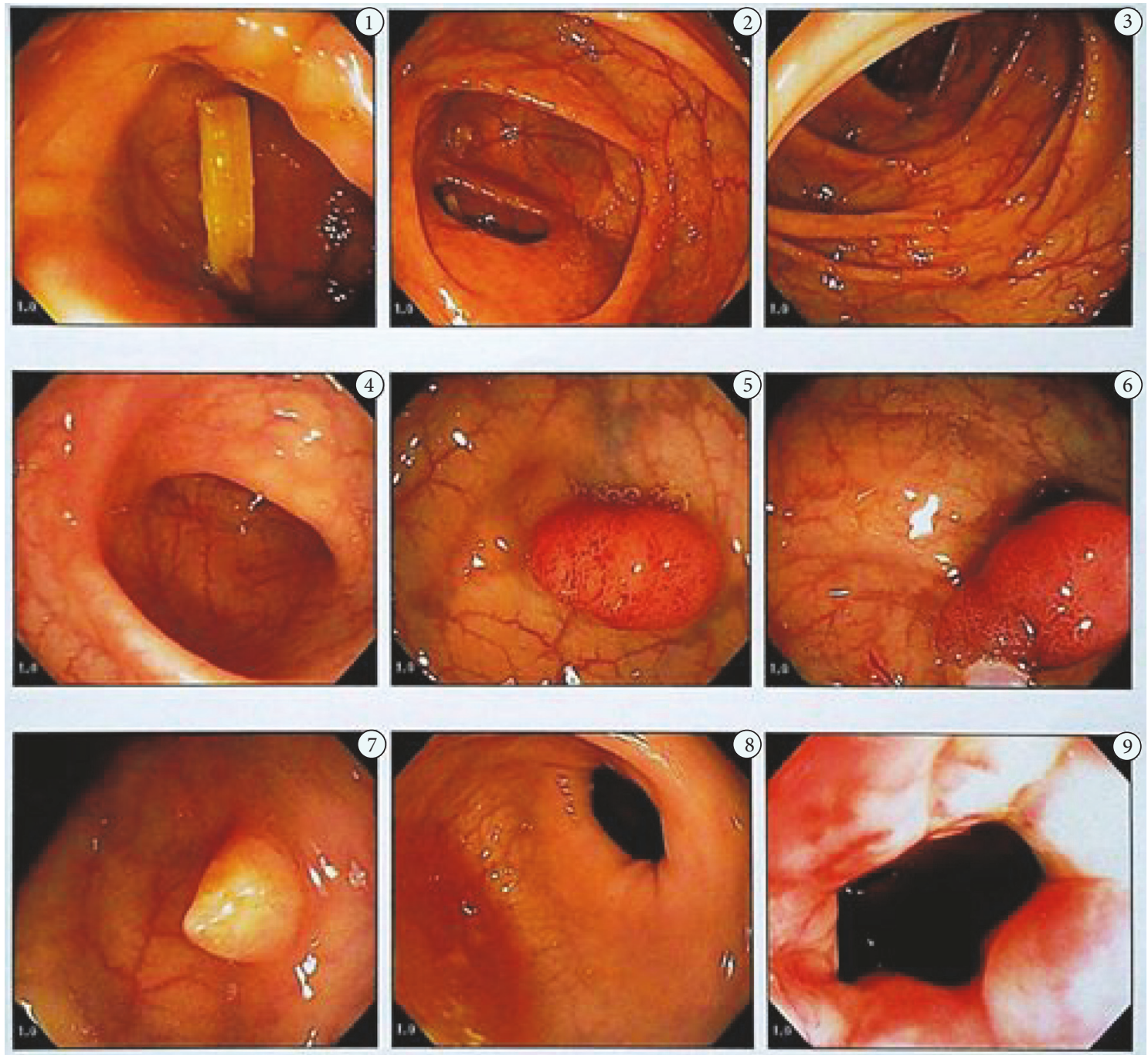

FIGURE 2: Images of videocolonoscopy that show the presence of polyps.

of cases in which there was a coexistence of intestinal disease and enterococcal endocarditis. Considering the inconclusive investigation of data so far, in order to be admissible to affirm a significant correlation between these two pathologies, it is important that further investigation with colonoscopy be performed in such cases, with the objective of elucidating the relevance of the association.

\section{Conflicts of Interest}

The authors declare that they have no conflicts of interest.

\section{References}

[1] E. Milbrandt, "A novel source of enterococcal endocarditis," Clinical Cardiology, vol. 21, no. 2, pp. 123-126, 1998.

[2] J. M. Pericàs, J. Corredoira, A. Moreno et al., "Relationship Between Enterococcus faecalis Infective Endocarditis and Colorectal Neoplasm: Preliminary Results From a Cohort of 154 Patients," Revista Española de Cardiología (English Edition), vol. 70, no. 6, pp. 451-458, 2017.
[3] J. Corredoira, M. J. Garcia-Pais, A. Coira, R. Rabunal, F. GarciaGarrote, J. Pita et al., "Differences between endocarditis caused by Streptococcus bovis and Enterococcus spp. and their association with colorectal cancer," European Journal of Clinical Microbiology \& Infectious Diseases: Official Publication of The European Society of Clinical Microbiology, vol. 34, pp. 1657-1665, Aug 2015.

[4] P. M. Miller, E. B. Frank, and R. A. Fischer, "Enterococcal endocarditis in association with cancer of the colon: report of a case and review of the literature," The Journal of the American Osteopathic Association, vol. 85, no. 11, pp. 751-753, 1985.

[5] R. Amarnani and A. Rapose, "Colon cancer and enterococcus bacteremia co-affection: a dangerous alliance," Journal of Infection and Public Health, 2017.

[6] C. Abeni, L. Rota, C. Ogliosi, P. Bertocchi, P. B. Centurini, and A. Zaniboni, "Correlation among Streptococcus bovis, endocarditis and septicemia in a patient with advanced colon cancer: a case report," Journal of Medical Case Reports, vol. 7, article no. 185, 2013.

[7] R. Mello, M. da Silva Santos, W. Golebiosvki, C. Weksler, and C. Lamas, "Streptococcus bovis endocarditis: analysis of cases 
between 2005 and 2014," Brazilian Journal of Infectious Diseases, vol. 19, no. 2, pp. 209-212, 2015.

[8] "AgudeloHiguita NI HMED,epidemiology, and implications for treatment," in Enterococci: From Commensals to Leading Causes of Drug Resistant Infection, M. S. Gilmore, D. B. Clewell, Y. Ike et al., Eds., vol. 4, Massachusetts Eye and Ear Infirmary, Boston, Mas, USA, 2014, https://www.ncbi.nlm.nih.gov/books/ NBK190429/.

[9] M. L. Fernández Guerrero, A. Goyenechea, C. Verdejo, R. F. Roblas, and M. De Górgolas, "Enterococcal endocarditis on native and prosthetic valves: a review of clinical and prognostic factors with emphasis on hospital-acquired infections as a major determinant of outcome," Medicine, vol. 86, no. 6, pp. 363-377, 2007.

[10] M. Ceci, G. Delpech, M. Sparo, V. Mezzina, S. S. Bruni, and B. Baldaccini, "Clinical and microbiological features of bacteremia caused by enterococcus faecalis," Journal of Infection in Developing Countries, vol. 9, no. 11, pp. 1195-1203, 2015.

[11] A. S. Abdulamir, R. R. Hafidh, and F. Abu Bakar, "The association of Streptococcus bovis/gallolyticus with colorectal tumors: the nature and the underlying mechanisms of its etiological role," Journal of Experimental \& Clinical Cancer Research: CR, vol. 30, 11 pages, 2011.

[12] C.-E. Tsai, C.-T. Chiu, C. K. Rayner et al., "Associated factors in Streptococcus bovis bacteremia and colorectal cancer," Kaohsiung Journal of Medical Sciences, vol. 32, no. 4, pp. 196-200, 2016.

[13] X. Wang, T. D. Allen, R. J. May, S. Lightfoot, C. W. Houchen, and M. M. Huycke, "Enterococcus faecalis induces aneuploidy and tetraploidy in colonic epithelial cells through a bystander effect," Cancer Research, vol. 68, no. 23, pp. 9909-9917, 2008.

[14] K. Shimizu, H. Ogura, T. Hamasaki et al., "Altered gut flora are associated with septic complications and death in critically ill patients with systemic inflammatory response syndrome," Digestive Diseases and Sciences, vol. 56, no. 4, pp. 1171-1177, 2011.

[15] N. Maharshak, E. Y. Huh, C. Paiboonrungruang et al., "Enterococcus faecalis gelatinase mediates intestinal permeability via protease-activated receptor 2," Infection and Immunity, vol. 83, no. 7, pp. 2762-2770, 2015.

[16] K. M. Van Der Heijden, I. M. Van Der Heijden, F. H. Galvao et al., "Intestinal translocation of clinical isolates of vancomycinresistant Enterococcus faecalis and ESBL-producing escherichia coli in a rat model of bacterial colonization and liver ischemia/reperfusion injury," PLoS ONE, vol. 9, no. 9, Article ID 0108453, 2014.

[17] C. J. O’Boyle, J. MacFie, C. J. Mitchell, D. Johnstone, P. M. Sagar, and P. C. Sedman, "Microbiology of bacterial translocation in humans," Gut, vol. 42, no. 1, pp. 29-35, 1998.

[18] M.-T. Liong, "Safety of probiotics: translocation and infection," Nutrition Reviews, vol. 66, no. 4, pp. 192-202, 2008. 


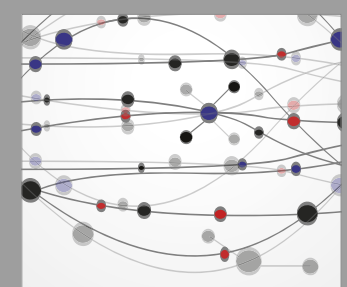

The Scientific World Journal
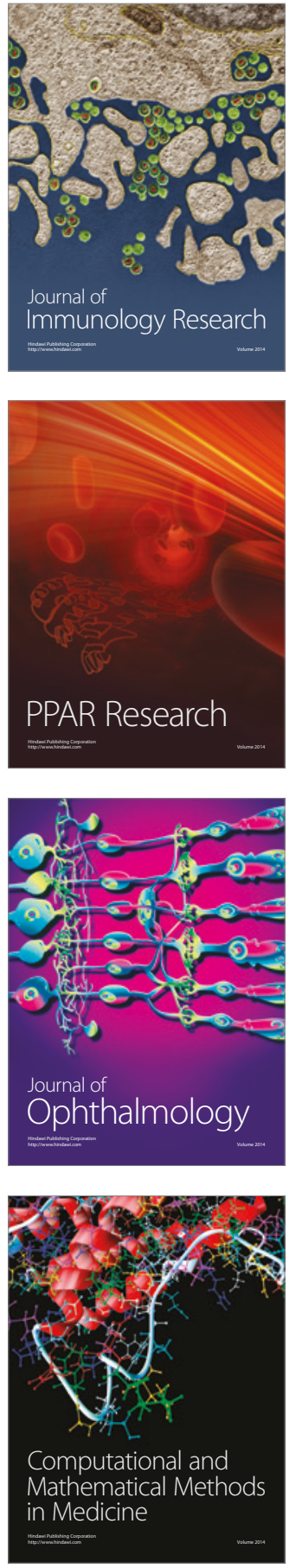

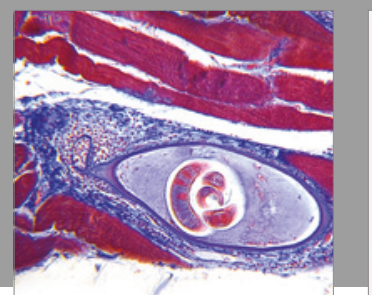

Gastroenterology Research and Practice
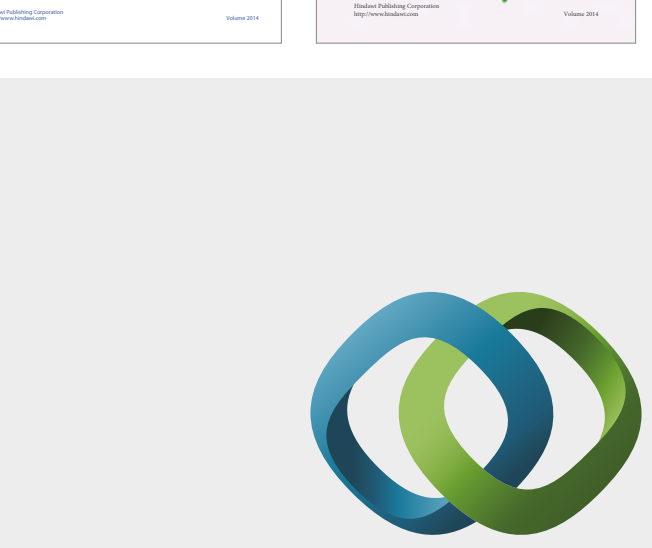

\section{Hindawi}

Submit your manuscripts at

https://www.hindawi.com
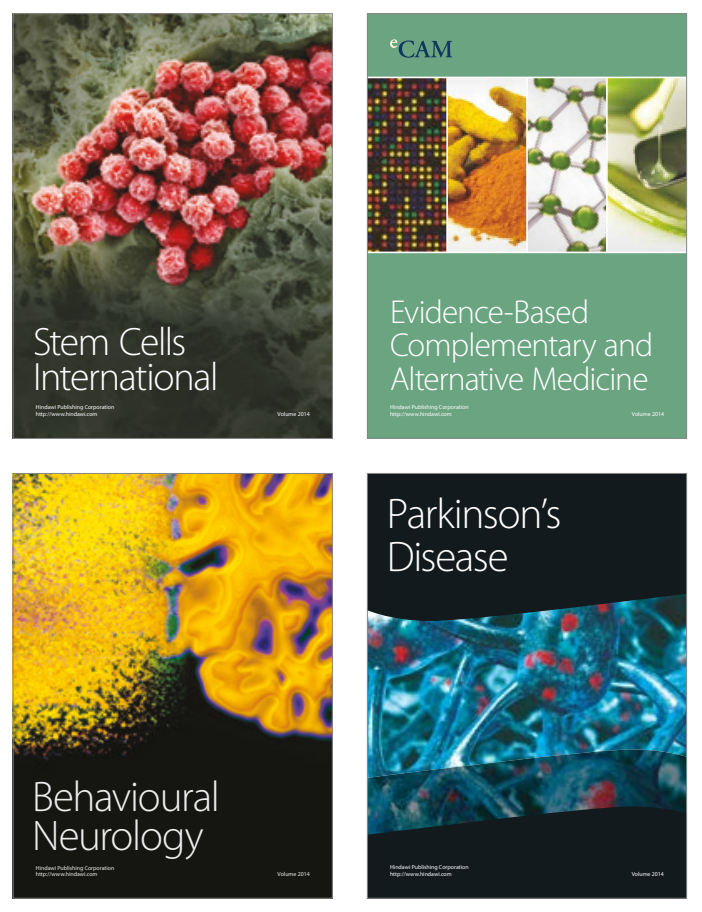
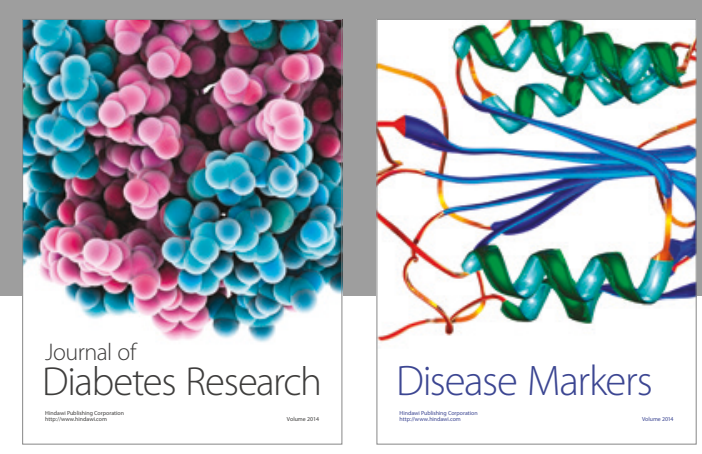

Disease Markers
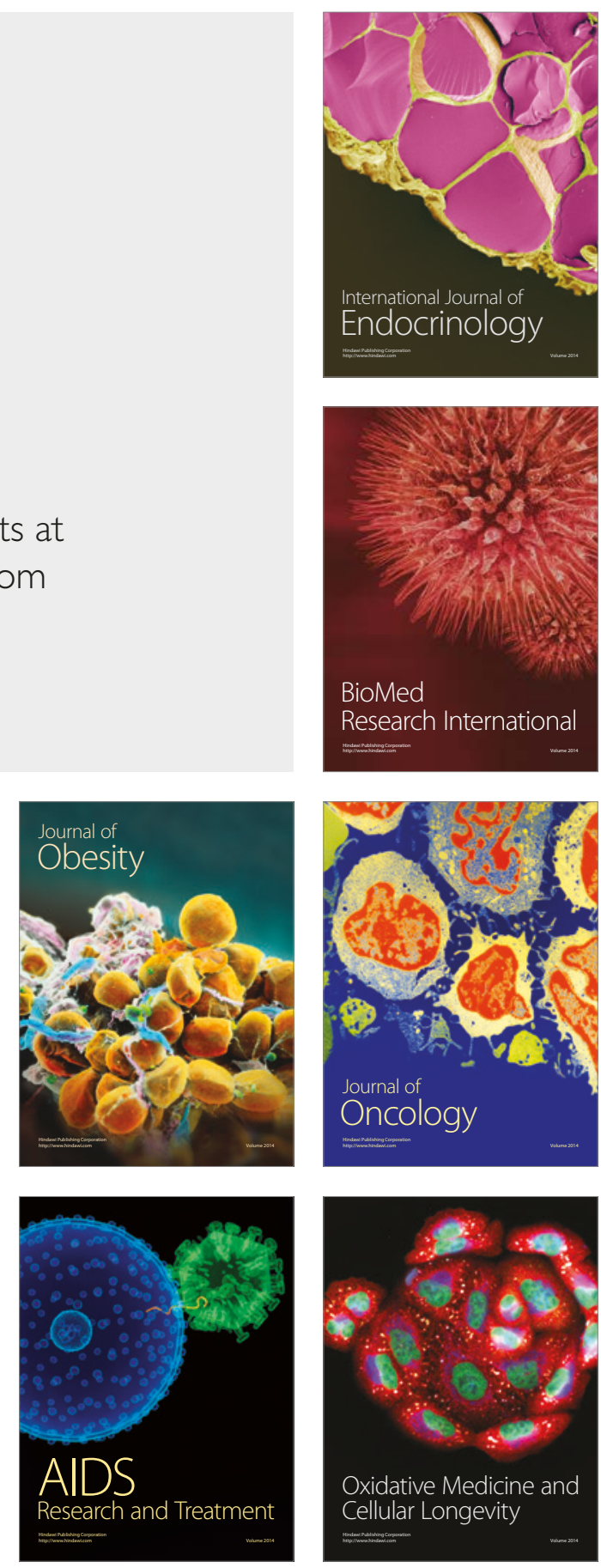